\title{
VERTICAL-CAVITY SURFACE-EMITTING SEMICONDUCTOR LASERS WITH DIFFUSED QUANTUM WELLS
}

\author{
C.W. Lo, S.F. Yu and E.H. Li \\ Department of Electrical and Electronic Engineering, The University of Hong Kong, Pokfulam Road, Hong Kong
}

\begin{abstract}
A self-consistent dynamic model is developed including the current distribution, carrier diffusion rate and spatial hole burning effects to investigate the modulation response of vertical-cavity surface-emitting lasers with diffused quantum wells structure. It is found that the overall performance including relaxation oscillation frequency and modulation bandwidth is improved.
\end{abstract}

\section{INTRODUCTION}

Vertical cavity surface emitting lasers (VCSELs) have the most attractive features such as extremely low threshold current, good beam quality and dynamic single mode performance. Therefore, VCSELs are the promișing light sources for optical fibre communications as well as optical interconnection systems. It is demonstrated that the maximum intrinsic relaxation oscillation frequency of VCSELs excess $70 \mathrm{GHz}$ [1]. This is because of the high photon density inside the laser cavity and short photon lifetime. However, the performance of VCSELs are limited by the confinement of radial optical field and current distribution. On the other hand, the concentration of impurity varies the refractive index and carrier diffusion rate of diffused Quantum Well (DFQW) material [2]. A defined pattern of refractive index profile can be obtained by selective area disordering of quantum well materials and this technique can be utilized for the fabrication of optical devices such as VCSELs [3]. In this paper, the characteristic of a VCSEL with DFQW structure which serves as the spacer and active regions is studied theoretically.

\section{DEVICE STRUCTURE}

The schematic of vertical-cavity surface-emitting laser with diffused Quantum Wells structure used in our calculation is shown in figure 1 . The device has a built-in index guided structure and a circular metal contact is formed on the epitaxial side ( $\mathrm{p}$-side) for current injection. The $\mathrm{Al}_{0.3} \mathrm{Ga}_{0.7} \mathrm{As} / \mathrm{GaAs}$ quantum well active layer is sandwiched between two undoped spacer layers and two Bragg reflectors, which provide optical feedback for lasing. The undoped spacer layers have thickness of half- wavelength each and the Bragg reflectors are formed by alternating layers of AlAs and AlGaAs with quarterwavelength thickness, and consists of 15 such pairs on both the $\mathrm{n}$ - and $\mathrm{p}$-side. The active region consists of six $\mathrm{Al}_{0.3} \mathrm{Ga}_{0.7} \mathrm{As} / \mathrm{GaAs}$ quantum wells with well width of $100 \AA$ and total thickness of half-wavelength. The corresponding longitudinal variation of effective refractive index profile of the entire multilayer VCSELDFQW structure is also shown in figure 1 . The device is assumed to be operated at $0.85 \mu \mathrm{m}$.

The confinement of radial optical field and carrier concentration can be achieved by interdiffusion of quantum well along the spacer and active layers as shown in figure 1 . The DFQW can be modeled by an error function and the extent of interdiffusion is characterized by a diffusion length, $L_{d}=\sqrt{ }(D t)$, where $D$ and $t$ are the diffusion coefficient and time, respectively. The details of these calculations can be found in references $4 \& 5$. A step change of $L_{d}$ along the radial direction forms the core and cladding regions of the VCSEL and the corresponding magnitudes of $L_{d}$ between the core and cladding regions are equal to 0 and $10 \AA$, respectively. The different of refractive index between the core and cladding regions is about 0.045 .

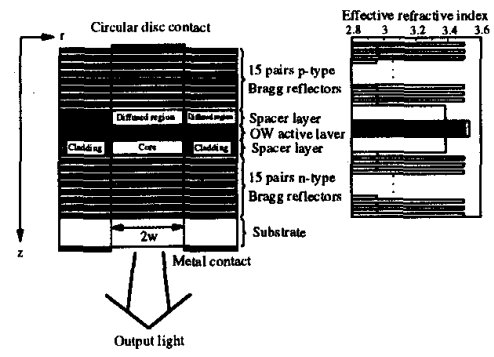

Figure 1. The schematic diagram of DFQWs-VCSEL structure.

\section{LASER MODEL}

The static and dynamic behaviour of DFQW-VCSEL is analyzed by our recently developed laser model [6]. The model takes into account the three dimensional variation of optical field distribution, current spreading and carrier spatial hole burning. The radial field 
distribution (in $r$ direction) is obtained by the wave equation and the corresponding optical field propagating along the longitudinal direction, $\mathrm{z}$, can be calculated by using the scattering matrix method [7]. The spatial carrier distribution and current spreading are determined by the van Roosbroeck semiconductor equations. The optical field distribution, carrier concentration and current profile inside the laser cavity are then computed in a selfconsistent manner.

In our analysis, the TE polarized optical gain, $g(\lambda)$, at room temperature is calculated by the density matrix approach. It is assumed that the propagation direction of the generated photon is parallel to the $\mathrm{QW}$ layers. At a particular $\mathrm{L}_{d}$ and at an external carrier injection level, $\mathrm{N}$, the TE net optical gain spectrum, G, can be approximated by $\mathrm{G}(\mathrm{N})=\mathrm{a}_{\mathrm{N}} \log \left(\mathrm{N}(\mathrm{r}, \mathrm{t}) / \mathrm{N}_{\mathrm{o}}\right)$, where $\mathrm{a}_{\mathrm{N}}$ is a fitted parameter and $\mathrm{N}_{\mathrm{o}}$ is the carrier concentration at transparency.

\section{RESULTS}

In the model, it is assumed that the value of $a_{N}$ and $N_{o}$ for the case $L_{d}=0 \AA$ are equal to $1300 \mathrm{~cm}^{-1}$ and $2.357 \times 10^{18} \mathrm{~cm}^{-3}$, and for the case of $L_{d}=10 \AA$ are equal to $1353 \mathrm{~cm}^{-1}$ and $2.269 \times 10^{18} \mathrm{~cm}^{-3}$, respectively. The core radius, $w$ is equal to $2.5 \mu \mathrm{m}$ and the total length of the laser is equal to $4.467 \mu \mathrm{m}$. The diffusion coefficient inside the diffused region is assumed equal to half of its original value. The injection current is assumed to be well confined within the core region. The dynamic response of DFQW-VCSELs are calculated for the steady state output power maintained at $0.25,1$ and $2 \mathrm{~mW}$, respectively. Figure 2 shows the distribution profiles of carrier concentration inside the active layer with three different steady state output powers. Spatial hole burning of carrier concentration is observed for the case of high injection current such that the steady state output power is equal to $2 \mathrm{~mW}$, however, high order mode (radial mode) is not excited under this situation. Furthermore, the improvement of relaxation oscillation frequency (ROF) can be explained by the increment of differential gain inside the active layer. This is because the differential gain, $a_{N} / N$, increases with the reduction of carrier concentration near the center of the core and hence the ROF is enhanced [8]. It also leads to the enhancement of the modulation bandwidth of the device (see figure 3). However, the carrier diffusion rate has less influence on the rolloff of the AM response.

\section{CONCLUSION}

In this paper, the improvement of ROF under the high power operation (at $2 \mathrm{~mW}$ ) is predicated theoretically by using the step change of interdiffusion length along the radial direction in VCSEL with DFQW active region. Results show that DFQW-VCSEL exhibit stable as well as single mode operation for the static situation under high current injection. In addition, DFQW device has an advantage over conventional VCSEL is that the confinement of radial optical field and current distribution can be achieved easily.

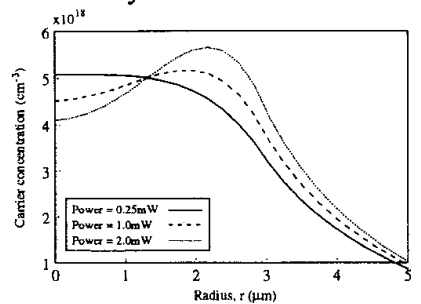

Figure 2. Distribution of carrier concentration along the active layer of DFQWs-VCSEL for different steady state output powers.

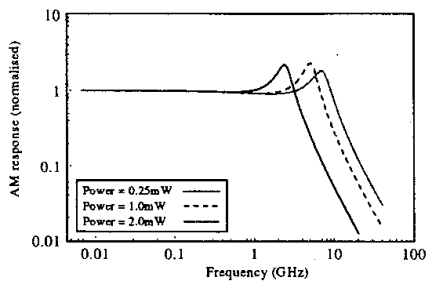

Figure 3. Amplitude modulation response of DFQWs-VCSEL for different steady state output powers.

\section{ACKNOWLEDGMENT}

This work is supported by the HKU-CRCG grants.

\section{REFERENCES}

[1] D. Tauber, G. Wang, R.s. Geels, J.E. Bowers and L.A. Coldern, 'Large and small signal dynamics of vertical cavity surface emitting lasers', Appl. Phys. Lett., 62, pp. 352-327, 1993

[2] E.H. Li and B.L. Weiss, 'Analytical solution of the absorption coefficients of AlGaAs-GaAs hyperbolic quantum wells', IEEE J. Quantum Electron., QE-29, pp. 311-321, 1993

[3] R.P. Bryan, J.J. Coleman, L.M. Miller, M.E. Givens, R.S. Averback and J.L. Klatt, 'Impurity induced disordered quantum well heterostructure stripe geometry lasers by MeV oxygen implantation', Appl. Phys. Lett., 55, pp.9496, 1989.

[4] E.H. Li and K.S. Chan, 'Laser gain and current density in a disordered $\mathrm{AlGaAs} / \mathrm{GaAs}$ quantum well', Electronics Lett., 29, pp.1233-1234, 1993.

[5] E.H. Li, B.L. Weiss and K.S. Chan, 'Effect of interdiffusion on the subbands in an $\mathrm{Al}_{\mathrm{x}} \mathrm{Ga}_{1-\mathrm{x}} \mathrm{As} / \mathrm{GaAs}$ single-quantum-well structure', Phys. Rev. B, 46, pp.15181-15192, 1992.

[6] S.F. Yu, 'Large signal dynamic model of Vertical cvity surface emitting laser', IEEE/LEOS '95, accepted for presentation.

[7] L.A. Coldren and T.L. Koch, 'Analysis and design of coupled cavity lasers-Part I: Threshold gain analysis and design guidelines', IEEE J. Quantum Electron., QE-20, pp.659-670, 1984.

[8] J. Hong, W.P. Huang and T. Makino, 'Effect of linear gain saturation on small-signal dynamics of MQW DFB lasers', IEEE Photon. Technol. Lett., 5, pp.1373-1376, 1993. 